\title{
Dopplerfluxometria do Ducto Venoso - Relação com a Gasometria em Fetos Prematuros com Centralização de Fluxo Sangüineo
}

\author{
Ductus Venosus Velocimetry: Relationship with Fetal Blood \\ Gases in Preterm Fetuses Presenting Brain Sparing Reflex \\ Renato Augusto Moreira de Sá, Hermógenes Chaves Netto \\ Laudelino Marques Lopes, Mário Jorge Viegas Barreto, Antônio Carlos Vieira Cabral \\ RESUMO
}

\begin{abstract}
Objetivo: avaliar o desempenho da dopplerfluxometria do ducto venoso (DV) na detecção da acidemia em fetos prematuros apresentando centralização de fluxo sangüineo.

Métodos: estudo observacional transversal. A população em estudo consistiu de 48 gestantes entre a $25^{a}$ e a $33^{a}$ semana de gestação, que apresentavam centralização de fluxo sangüineo fetal (relação umbílico/cerebral $>1$ ). O tempo decorrido entre o diagnóstico de centralização fetal e o nascimento (cesariana sob anestesia peridural) foi de até cinco horas. Os seguintes parâmetros foram estudados: relação sístole ventricular atrial ( $\mathrm{S} / \mathrm{A})$ do ducto venoso, $\mathrm{pH}$ e base excess $(B E)$ de amostra de sangue da veia umbilical colhida imediatamente após o nascimento. A relação S/A do DV foi considerada anormal quando maior que 3,6. Os conceptos foram classificados quanto ao resultado da gasometria, sendo considerada gasometria anormal quando $\mathrm{pH}<7,26$ e $B E<6 \mathrm{mmol} / \mathrm{L}$. O estudo estatistico utilizado foi o teste de Fisher, considerado significativo quando $p<0,05$.

Resultados: a correlação entre a gasometria do cordão umbilical de fetos prematuros centralizados e a relação $S / A$ do $D V$ foi significativa ( $p=0,0000082$; teste de Fisher). A dopplervelocimetria do DV foi capaz de identificar 10 dos 14 fetos com gasometria anormal. Por outro lado, 32 dos 34 fetos com gasometria normal foram corretamente identificados. A sensibilidade da dopplerfluxometria do DV para o diagnóstico de acidemia fetal ao nascimento foi de 71\%, a especificidade foi de 94\%, a taxa de falso-negativo de 8\%, a taxa de falsopositivo de 4\%, o valor preditivo positivo de $83 \%$ e o valor preditivo negativo de $89 \%$. A probabilidade pré-teste para um feto centralizado de apresentar acidemia ao nascimento foi de $31 \%$. Já a probabilidade de acidemia após resultado do DV anormal (probabilidade após teste anormal) foi de 84\%, ao passo que a probabilidade de acidemia fetal com o DV normal (probabilidade após-teste normal) foi de apenas $10 \%$.

Conclusão: a análise da relação $S$ / A do ducto venoso se mostra adequada para o diagnóstico de gasometria anormal em fetos prematuros, centralizados.
\end{abstract}

PALAVRAS-CHAVE: Dopplervelocimetria. Gasometria, Sofrimento fetal.

\section{Introdução}

A expectativa para a sobrevivência neonatal é influenciada pela maturidade e pela idade gestacional. A imprecisão desta última dificulta a avaliação do potencial de sobrevida. Assim sendo,

Correspondência:

Renato Augusto Moreira de Sá

Rua das Laranjeiras 445, sub-solo - Laranjeiras

22240-002 - Rio de Janeiro - RJ a maioria dos dados neonatais se baseia no peso ao nascimento ${ }^{1}$.

A repercussão fetal das agressões por ele sofridas, extrinsecas ou intrinsecas, depende da época e da intensidade em que estas agressões ocorreram. Desde há muito é sabido que quanto mais precoce for a ofensa, mais grave serão suas conseqüências para o concepto, resultando em aumento da mortalidade e da morbidade. Outro fator importante é o impacto econômico causado pela prematuridade. Estima-se que um terço da verba 
destinada à saúde para o primeiro ano de vida seja gasto com fetos prematuros abaixo de $1.500 \mathrm{~g}$, que correspondem a 1,5\% dos nascimentos nos Estados Unidos ${ }^{1}$.

A probabilidade de óbito neonatal é extremamente elevada quando o parto ocorre até a $25^{a}$ semana (acima de 80\%) e apresenta queda significativa a cada semana até a $33^{a}$ semana de gestação. Quando se analisa a morbidade, as maiores taxas ocorrem em conceptos nascidos com peso entre 500 e $750 \mathrm{~g}$, caindo progressivamente a cada $250 \mathrm{~g}^{2}$.

Detectar as condições intra-uterinas normais e as modificações encontradas em suas eventuais alterações patológicas tem sido a preocupação constante dos pesquisadores. A natural limitação imposta à investigação em humanos e a relativa inacessibilidade do feto constituem os principais obstáculos a estas pretensões.

Em 1977, Fitzgerald e Drumm relataram pela primeira vez o uso da dopplerfluxometria no rastreamento do bem-estar fetal ${ }^{3}$. Desde então foram se acumulando dados que demonstraram associações estatisticamente significativas entre o sonograma anormal do Doppler da circulação fetoplacentária e complicações gravídicas ${ }^{4}$.

Este método não invasivo, rápido e seguro contribuiu para a elucidação dos conceitos fisiológicos da hemodinâmica materna e fetal e admitiu novos modelos para explicar aspectos hemodinâmicos antes inteiramente ignorados pela obstetrícia.

A centralização passou a ser reestudada de modo mais preciso e não invasivo, permitindo caracterizar os primeiros sinais defensivos fetais em face do sofrimento intra-uterino ${ }^{5}$.

A dopplerfluxometria permitiu ainda a melhor compreensão da função cardiaca nos fetos com crescimento intra-uterino retardado (CIUR), que apresentam aumento das propriedades de enchimento ventricular com diminuição da relação entre o pico correspondente ao enchimento ventricular rápido (E) e a sístole atrial (A), ou seja, a diminuição da relação E/A nas válvulas átrioventriculares ${ }^{6}$, menor velocidade de pico (VP) na aorta e artéria pulmonar ${ }^{7}$, aumento do tempo de velocidade de pico na artéria aorta e diminuição na pulmonar ${ }^{8}$ e relativo aumento do débito cardíaco esquerdo associado ao decréscimo à direita ${ }^{9}$. Estas alterações hemodinâmicas intracardiacas são compatíveis com desvio preferencial do débito cardíaco em favor do ventrículo esquerdo para melhorar a perfusão cerebral. Entretanto, nos estágios iniciais da doença, o suprimento de oxigênio e outros substratos podem se manter próximos da normalidade, apesar da absoluta redução da troca placentária ${ }^{10}$.
Estudos longitudinais em fetos com CIUR e deterioração progressiva têm ajudado a elucidar a história natural destas modificações hemodinâmicas durante a insuficiência úteroplacentária ${ }^{11}$. Estes estudos demonstraram que tanto o tempo de velocidade de pico nas artérias aorta e pulmonar como as taxas de débito ventricular direito-esquerdo se mantiveram estáveis durante os registros seriados. Estes achados são consistentes com a ausência de outras alterações significativas na resistência periférica e com a redistribuição do débito cardíaco após o estabelecimento da centralização. Em fetos com CIUR gravemente acometidos, entretanto, a velocidade de pico e o débito cardíaco declinam gradualmente, sugerindo deterioração progressiva da função cardiaca. Como conseqüência, o enchimento cardíaco aumenta ${ }^{7}$.

Estudos da circulação venosa fetal demonstraram que aumento do fluxo reverso na veia cava inferior durante a contração atrial ocorre quando existe agravamento da condição fetal, o que sugere alto gradiente de pressão no átrio direito. O próximo passo da doença é a ampliação do fluxo reverso na veia cava e ducto venoso, levando ao aumento da relação sístole ventricular/sístole atrial (S/A) pela redução do componente A das ondas de Doppler ${ }^{11}$. Finalmente, a alta pressão venosa induz a redução da velocidade no final da diástole refletida na veia umbilical como pulso ${ }^{12}$.

Warshaw $^{13}$ questionou se o CIUR decorrente do suprimento restrito de nutrientes representava doença ou adaptação favorável ao feto e enfatizava que a diminuição do tamanho do feto, o desvio preferencial da circulação para órgãos nobres (centralização), a aceleração da maturidade pulmonar e a policitemia representariam estratégia inicial de adaptação que só se tornaria patológica quando a privação é extrema. O estudo dos vasos venosos indicaria este estado de descompensação por apresentar melhor correlação com o estado ácido-básico fetal. Em outras palavras, a acidemia poderia ser detectada pela dopplerfluxometria venosa. A maioria dos estudos disponiveis usou gasometria de cordão obtido por cordocentese, entretanto o procedimento invasivo é fator de estresse fetal e por sua vez altera sua hemodinâmica. Além disso, as condutas neonatais baseiam-se na gasometria do cordão obtida ao nascimento.

Dos diversos métodos propedêuticos investigados com o objetivo de avaliar a saúde do concepto, nenhum foi completamente bem sucedido na identificação do sofrimento fetal crônico, que se caracteriza por modificações bioquímicas do meio interno, ocasionadas pela redução dos intercâmbios feto-maternos e que suscitam no feto reações compensatórias. 
O risco da prematuridade deve ser comparado ao da acidemia grave, ambas apresentando elevados indices de mortalidade perinatal e morbidade em longo prazo. Daí a importância de se conhecer com precisão a idade gestacional antes da conduta obstétrica ${ }^{14}$.

O objetivo do estudo é avaliar o desempenho da dopplerfluxometria do ducto venoso na detecção da acidemia em fetos prematuros apresentando centralização de fluxo sangüíneo.

\section{Pacientes e Métodos}

O estudo realizado foi do tipo observacional transversal para avaliação de teste de diagnóstico. Os dados foram colhidos prospectivamente e a amostragem foi feita de forma aleatória.

Todas as pacientes participantes do estudo foram informadas sobre os objetivos, segurança e os tipos de exames propostos para a pesquisa. $\mathrm{O}$ consentimento pós-informação foi obtido sem formular-se por escrito, de acordo com o artigo $13^{\circ}$, capítulo II da resolução número $01 / 88$ do Conselho Nacional de Saúde (CNS), por se tratar de pesquisa com risco mínimo (artigo $7^{\circ}$, parágrafo II, capítulo I da resolução 01/88 do CNS).

Foi considerado como critério de inclusão a idade gestacional correta - todas as gestantes selecionadas apresentavam idade gestacional calculada em semanas completas pela data da última menstruação, segundo critério adotado pela Organização Mundial de Saúde ${ }^{15}$ e referendado pela FIGO em $1976^{15}$. A idade gestacional foi confirmada com exames ultra-sonográficos no primeiro e segundo trimestres, com o uso dos seguintes parâmetros: comprimento cabeçanádega, diâmetro biparietal e comprimento do fêmur. Todas as pacientes foram submetidas a cesariana sob anestesia peridural na ausência de trabalho de parto, tendo como indicação básica para o ato cirúrgico a pré-eclampsia ou a centralização fetal. Não houve influência do resultado da dopplerfluxometria do ducto venoso na indicação da cesariana, por não se tratar de exame de rotina, e os médicos que assistiam às pacientes não eram informados do resultado da dopplerfluxometria do ducto venoso. Foram considerados como critérios de exclusão: a presença de anormalidades anatômicas identificadas à ultrasonografia, a gestação múltipla e a colheita inadequada da amostra de sangue da veia umbilical para análise gasométrica após o nascimento.

Inicialmente realizou-se o perfil hemodinâmico fetal (dopplerfluxometria arterial, usando como referência a artéria cerebral média e a ar- téria umbilical) em todas as pacientes. A relação umbílico/cerebral (U/C) fetal foi definida pela razão entre o fluxo da artéria umbilical e da artéria cerebral média. A centralização fetal foi caracterizada quando a razão entre o índice de pulsatilidade da artéria umbilical (U) e da artéria cerebral média (C) era maior ou igual a 1 (U/C >1). A seguir foi realizada a dopplerfluxometria do ducto venoso.

Os exames de dopplerfluxometria foram realizados utilizando-se aparelho (modelo SSH - 140A; Toshiba Corporation, Japão) com transdutor convexo de 3,75 $\mathrm{MHz}$. Todas as medidas de dopplerfluxometria foram feitas na ausência de contrações uterinas e em período de absoluto repouso fetal, aí se incluindo os movimentos respiratórios. Em todos os exames o filtro do aparelho foi ajustado para sensibilidade de $100 \mathrm{~Hz}$. Foram determinados os pontos A (pico da sístole) e B (final da diástole), obtendo-se automaticamente as relações sístole/diástole (A/B), indice de resistência e índice de pulsatilidade, para as artérias umbilical e cerebral média.

Para obtenção do sonograma da artéria cerebral média pela via transabdominal, realizavase secção horizontal do cérebro, incluindo o tálamo e o cavum do septo pelúcido (plano de corte habitual para a medida do diâmetro biparietal). Movendo-se o transdutor caudalmente em plano paralelo ao anterior em direção à base do crânio, foram identificadas as fossas anterior, média e posterior. As artérias cerebrais eram vistas com auxílio do Doppler colorido como duas estruturas pulsando em estreita proximidade das asas maiores do esfenóide. Acionamos o Doppler pulsátil para obtenção das ondas de velocidade e realizamos as medições.

O sonograma da artéria umbilical foi obtido colocando-se o transdutor na parede abdominal materna e localizando-se com auxilio do Doppler colorido os três segmentos do cordão umbilical: proximal (próximo à inserção na parede abdominal fetal), alça livre e distal (próximo à inserção placentária). Após acionarmos o Doppler pulsátil, colocamos a janela de modo que a veia umbilical fosse visualizada no mesmo corte, obtivemos as ondas de velocidade para cada segmento e realizamos as medições. Caso houvesse diferença do indice de pulsatilidade nos três segmentos, considerávamos a média destes para a relação U/C.

O ducto venoso foi identificado usando a imagem de ultra-som em duas dimensões e Doppler colorido. A primeira etapa foi a localização do sinus portal e da inserção do cordão umbilical na parede abdominal, visualizados em corte de secção transversal do abdome fetal ou por vezes médio-sagital. Sem perder esta visão, o transdutor era movido até que a origem do ducto venoso no sinus portal fosse identificada. A janela 
do Doppler pulsátil era então posicionada na origem do vaso (istmo) e por vezes em sua porção distal $^{16}$ e obtidas as ondas de velocidade. Foram medidas a velocidade de pico durante a sístole ventricular (S) e a velocidade correspondente à contração atrial (A) e calculada a relação $\mathrm{S} / \mathrm{A}^{17}$. Para análise dos resultados foi usado como referência a curva de normalidade por nós desenvolvida. A média da relação $\mathrm{S} / \mathrm{A}$ considerada normal foi de $2,37+0,68$. Os valores extremos foram: percentil $5=1,5$ e percentil $95=3,6^{17}$. Os resultados foram classificados como anormais quando eram superiores aos valores da média mais dois desvios padrão da média, ou seja, maiores do que $3,6^{18}$.

O tempo decorrido entre o diagnóstico de centralização fetal e o nascimento (cesariana sob anestesia peridural) foi de até cinco horas. Para a colheita da amostra do sangue do cordão foram utilizadas seringas plásticas de $3 \mathrm{~mL}$ da marca Becton Dickinson (B-D) e agulha descartável da mesma marca (30 x 7 - 22G). Foi utilizada heparina sódica em ampolas de 0,25 mL em solução aquosa. A heparina foi aspirada para a seringa $e$ homogeneamente distribuida pelas paredes da mesma. A seguir expirava-se o conteúdo da seringa com a agulha voltada para cima, para expelir o ar remanescente (por meio desta técnica aproximadamente $0,2 \mathrm{~mL}$ da heparina permanecem na seringa e na agulha).

Imediatamente após o parto um segmento de cordão de aproximadamente 20 a 30 cm de comprimento era isolado e ocluído com duas pinças. A amostra de sangue $(2 \mathrm{~mL})$ era então colhida através da punção da veia umbilical com a seringa previamente preparada, sendo ocluída a seguir. As amostras foram enviadas para análise gasométrica em 10 minutos, em média, devidamente acondicionadas em isopor com gelo. A análise do sangue foi feita pelo aparelho Radiomiter ABL-5.

$\mathrm{O}$ resultado do $\mathrm{pH}$ foi analisado segundo os seguintes parâmetros: considerou-se acidemia se o $\mathrm{pH}$ do sangue da veia umbilical fosse inferior a 7,26 . A acidemia metabólica foi definida quando base excess $(\mathrm{BE})<6 \mathrm{mmol} / \mathrm{L} \mathrm{e}_{\mathrm{pCO}}<50 \mathrm{mmHg}$, a acidemia respiratória quando $\mathrm{BE}>6 \mathrm{mmol} / \mathrm{L}$ e $\mathrm{pCO}_{2}$ $>50 \mathrm{mmHg}$ e a acidemia mista quando $\mathrm{BE}<6$ $\mathrm{mmol} / \mathrm{L} \mathrm{e} \mathrm{pCO}_{2}>50 \mathrm{mmHg}^{19}$. Para efeito de análise estatística foram consideradas como gasometria anormal aquelas que apresentavam acidemia metabólica e mista, uma vez que estão associadas a aumento significativo de complicações neonatais, diferente da acidemia respiratória, que não apresenta a mesma associação ${ }^{19}$. Foram considerados como pequenos para a idade gestacional aqueles fetos que apresentavam ao nascimento peso inferior ao percentil 10 do esperado para a idade gestacional.
Inicialmente calculamos o tamanho amostral com auxílio do programa computacional Epi-Info versão 5.01 de março de 1991. Fixou-se o erro alfa (probabilidade de resultado "falso-positivo") em 5\% (intervalo de confiança de 95\%) e o erro beta (probabilidade de resultado "falso-negativo") em 20\% (poder do estudo de 80\%). A razão dopplerfluxometria de ducto venoso normal/ dopplerfluxometria de ducto venoso alterado considerada foi de $7: 3$. A freqüência esperada de fetos acidóticos no grupo de dopplerfluxometria venosa normal foi de $20 \%$ e no grupo de dopplerfluxometria venosa comprometida foi de $90 \%$. Considerando estes parâmetros, o cálculo do tamanho amostral indicava a inclusão de 20 casos (14 normais e 6 alterados). Foram selecionadas para o estudo 48 gestantes com fetos centralizados, sendo 12 com Doppler de ducto venoso comprometido e 36 normais.

Para possibilitar a análise dos dados foram cotejados os resultados da dopplerfluxometria do ducto venoso (normal ou alterado) com os resultados da gasometria do cordão umbilical (normal ou anormal) por meio da tabela $2 \times 2$.

$\mathrm{O}$ estudo estatístico utilizado para analisar as variáveis categóricas (teste de significância da associação ou dependência) foi o teste exato de Fisher, já que o valor esperado em uma das caselas foi menor do que 5 . O resultado foi considerado significativo quando $\mathrm{p}<0,05$

Ainda foram calculados: sensibilidade, especificidade, taxa de falso-negativo, taxa de falso-positivo, valor preditivo dos resultados comprometidos, valor preditivo dos resultados normais, probabilidade pré-teste, razão de verossimilhança para a dopplerfluxometria anormal, razão de verossimilhança para a dopplerfluxometria normal, probabilidade após-teste anormal e probabilidade após-teste normal.

\section{Resultados}

Foram selecionadas 48 grávidas no período de janeiro de 1998 a setembro de 2000. A média das idades maternas foi de 29 anos (+ 1,8 anos). No momento do estudo a idade gestacional variou entre 25 e 33 semanas, média de 29,7 semanas (+ 1,8 semanas) e mediana de 30 semanas.

Todos os fetos apresentavam-se centralizados, dentre eles 12 com dopplerfluxometria de ducto venoso anormal e 36 com dopplerfluxometria normal. A prevalência de resultados de fetos com dopplervelocimetria de ducto venoso alterado no material estudado foi de $25 \%$.

A média de peso dos conceptos foi de $943 \mathrm{~g}$ ( $^{+}$ $232 \mathrm{~g})$. A taxa de fetos pequenos para a idade gestacional em nossa casuística foi de 79,2\%. 
A prevalência de acidemia metabólica/mista (BE anormal) foi de $29 \%$ na população estudada. A gasometria do cordão umbilical mostrou que nos fetos com ducto venoso anormal a acidemia foi mais prevalente do que naqueles com ducto venoso normal. A mediana do $\mathrm{pH}$ nos conceptos com ducto venoso anormal foi de $7,14(+0,08)$, ao passo que nos que apresentavam relação S/A do ducto venoso normal a mediana do $\mathrm{pH}$ foi de 7,26 (+ 0,05).

Analisando-se o gráfico de dispersão, observa-se que a grande maioria dos fetos que apresentavam acidemia ao nascimento se concentrava na faixa de normalidade da relação $\mathrm{S} / \mathrm{A}$ e os que apresentam gasometria anormal encontravam-se, em maior número, acima do percentil 95 (Figura 1).

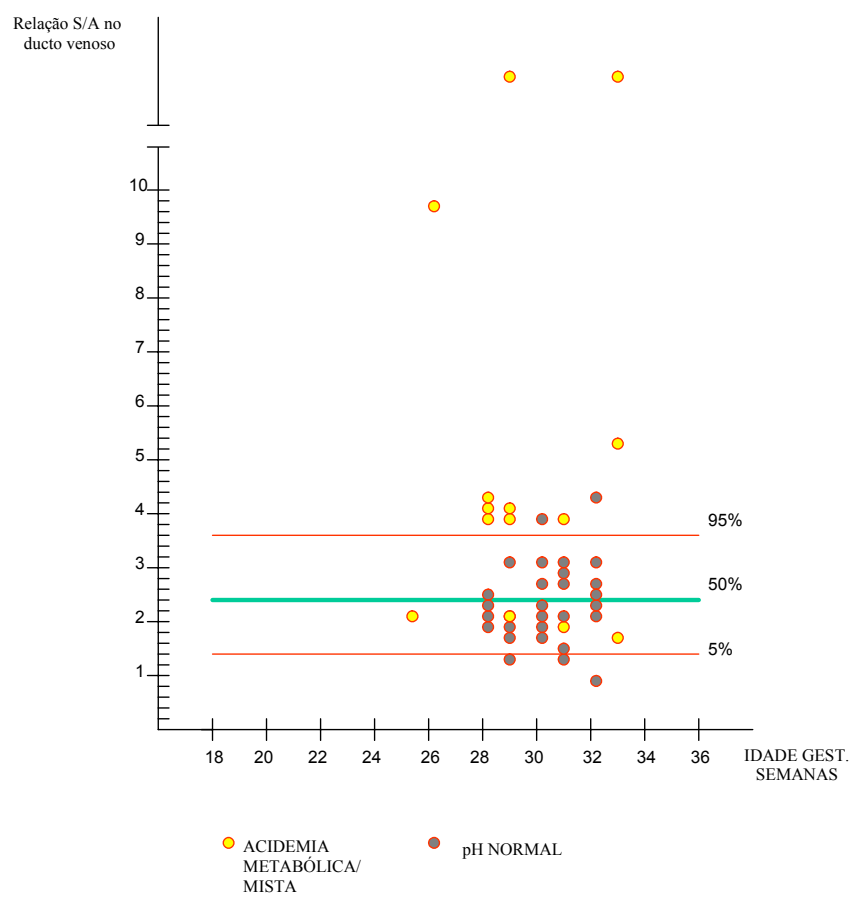

Figura 1 - Distribuição da casuística por idade gestacional em semanas, pelos valores de $\mathrm{pH}$ e relação sístole ventricular/atrial no ducto venoso.

Observou-se associação significativa entre o ducto venoso anormal e gasometria anormal ao nascimento ( $p=0,0000082$; teste de Fisher). Dentre os conceptos que apresentaram gasometria anormal $(n=14), 71,4 \%$ apresentaram dopplerfluxometria de ducto venoso anormal $(n=10)$ e $28,6 \%$, dopplerfluxometria de ducto venoso dentro da faixa de normalidade $(n=4)$. Dos 34 fetos que apresentaram gasometria normal, 94,1\% apresentaram dopplerfluxometria de ducto venoso normal $(n=32)$ e 5,9\%, dopplerfluxometria de ducto venoso anormal $(n=2)$. A sensibilidade da dopplerfluxometria do ducto venoso para o diagnóstico de acidemia fetal ao nascimento foi de $71 \%$, a especificidade foi de 94\%, a taxa de falso-negati- vo de $8 \%$, a taxa de falso-positivo de $4 \%$, o valor preditivo positivo de $83 \%$ e o valor preditivo negativo de $89 \%$.

A probabilidade (pré-teste) de um feto centralizado apresentar acidemia ao nascimento foi de $31 \%$. Já a probabilidade de acidemia após resultado do ducto venoso anormal (probabilidade após-teste anormal) foi de $84 \%$, para uma razão de verossimilhança de 12,14 . Já a probabilidade de acidemia fetal com o ducto venoso normal (probabilidade após-teste normal) foi de apenas 10\%, para uma razão de verossimilhança de 0,3.

A interpretação destes valores nos permite considerar que a probabilidade que um feto centralizado tem de apresentar gasometria alterada independente do resultado do ducto venoso é de $31 \%$. Uma vez que a relação $\mathrm{S} / \mathrm{A}$ do ducto venoso é superior a 3,6, a probabilidade de a gasometria estar alterada passa a ser de 84\%. Em contrapartida, na presença da relação S/A normal $(<3,6)$, a probabilidade de a gasometria estar alterada é de apenas $10 \%$.

\section{Discussão}

A análise do território venoso pode ser útil se o desejado é aguardar a maturidade ou reduzir a morbidade quando estamos lidando com prematuridade extrema. A mortalidade neonatal global diminui com o aumento do peso ao nascimento ${ }^{20}$. Baschat et al. ${ }^{21}$ consideram que a idade gestacional é o parâmetro que apresenta maior impacto nos resultados pós-natais.

A dopplervelocimetria venosa alterada parece indicar deterioração fetal em meio intrauterino inóspito no qual o feto não parece ter condição de sobreviver por muito tempo; precede as alterações de outros parâmetros biofísicos, como por exemplo as alterações da cardiotocografia, podendo ser parâmetro a se considerar isoladamente na decisão da interrupção da gestação ${ }^{22}$.

Dois mecanismos parecem ser responsáveis pelo padrão anormal das ondas de velocidade venosas: uma é o aumento da pós-carga ventricular direita e outra, a falência miocárdica ${ }^{23}$. Tem sido demonstrado que a hipoxemia crônica está relacionada ao aumento do fluxo miocárdico. A capacidade do feto de compensar a redução do fluxo placentário por meio da centralização está relacionada à adequada oxigenação miocárdica, uma vez que a pós-carga está aumentada. Neste tempo, embora exista aumento da resistência na circulação feto-placentária, a centralização se dá sem que haja alteração nas ondas de Doppler venosas. As alterações progressivas na circulação venosa indicam falência no mecanismo compensatório e 
o desenvolvimento de insuficiência cardiaca por hipoxia miocárdica ${ }^{24}$.

As pesquisas nesta área se justificam pelo fato de não sabermos por quanto tempo se deve monitorar um feto centralizado até que ele apresente sinais de comprometimento grave e se vale a pena investir tanto dinheiro e tempo em aparelhos médicos e pesquisa, se não formos capazes de atuar antes que o sofrimento fetal ocorra ${ }^{17}$. O risco de lesão fetal secundária à deficiência de oxigênio e nutrientes precisa ser comparado ao risco atribuído à prematuridade, que pode ser um grave problema antes de 32 semanas de gestação. Fetos pequenos para a idade gestacional parecem ser capazes de tolerar hipoxemia crônica sem lesão, permitindo que o parto se dê mais tardiamente pela redistribuição do fluxo (centralização), desde que a avaliação doplervelocimétrica venosa seja normal $^{23}$.

Hofstaetter et al. ${ }^{25}$ concluíram que velocidades anormais no ducto venoso são marcadores eficazes para resultados perinatais adversos. Baschat et al. ${ }^{21}$ demonstraram, por análise multivariada, que só a idade gestacional apresenta correlação com complicações pós-natais, ao passo que a dopplerfluxometria venosa anormal só estava associada à morte intra-útero. Entretanto, Ozcan et al. ${ }^{26}$ observaram que a dopplervelocimetria anormal do ducto venoso foi o único parâmetro que apresentou associação com mortalidade perinatal e índice de Apgar abaixo de 5 .

Com o propósito de testarmos se a alteração do fluxo no ducto venoso estava relacionada à acidemia fetal no momento do nascimento, decidimos realizar este estudo. A grande maioria dos ensaios que se propuseram a correlacionar a dopplervelocimetria à gasometria usaram a amostra de sangue obtida por cordocentese. Os efeitos de procedimentos invasivos no fluxo do ducto venoso têm sido examinados em fetos com crescimento adequado e CIUR, e comprovou-se que após a colheita da amostra por cordocentese a relação $\mathrm{S} / \mathrm{A}$ cai significativamente ${ }^{22}$. Pode ser ainda observada dupla pulsação na veia umbilical e incisura pós-sistólica na artéria umbilical após cordocentese em fetos gravemente comprometi$\operatorname{dos}^{27}$. Estas alterações hemodinâmicas podem explicar os altos índices de complicação da cordocentese neste grupo de pacientes ${ }^{23}$, motivo pelo qual optamos por não utilizar tal método de colheita.

A escolha do padrão de excelência se deveu ao fato de que Vintzileos et al. ${ }^{19}$ demonstraram que as acidemias metabólica e mista, em amostras de sangue de cordão ao nascimento, estavam associadas a aumento significativo de complicações neonatais, e que os valores da gasometria não se alteravam com a idade gestacional. A determinação bioquímica do equilíbrio ácido-básico fetal e neonatal é considerada padrão de excelência para diagnóstico de asfixia perinatal, importante na tomada de decisão da conduta neonatal. A acidemia metabólica fetal é parte terminal da via que leva os fetos com CIUR ao óbito. É objetivo primário dos testes anteparto a identificação precoce dos fetos acidóticos, visando previnir a mortalidade e o risco de lesão neurológica grave ${ }^{6}$. Clyman e Heymann $^{28}$ acreditam ser o sangue da veia umbilical o que melhor se presta para a avaliação fetal, uma vez que este atingirá diretamente o cérebro e o coração, não tendo sofrido mistura com sangue de retorno pouco oxigenado.

Em fetos de ovelhas submetidos à hipoxemia, a porcentagem de sangue proveniente da veia umbilical que é desviado do figado para o ducto venoso se eleva, aumentando a contribuição ao débito cardíaco. Isto resulta em maior aporte de sangue oxigenado da veia umbilical para o miocárdio e cérebro, embora a contribuição da hipoxemia isoladamente para a alteração de fluxo no ducto venoso seja pequena ${ }^{29}$. A maioria dos estudos conclui que se a hipoxemia estiver associada à acidemia ocorrerá redistribuição do sangue para o ducto venoso em detrimento do fluxo hepático ${ }^{23}$. Tais fatos nos fizeram optar pelo estudo do ducto venoso como parâmetro para identificar os fetos com acidemia.

O aumento da pressão no final da diástole no ventrículo direito não altera a velocidade de fluxo no ducto venoso, pois o sangue é dirigido preferencialmente para o ventrículo esquerdo através do forame oval. Durante a contração atrial, entretanto, o forame oval encontra-se fechado e podemos perceber a redução do fluxo ou fluxo reverso durante a contração atrial à proporção que aumenta a resistência periférica e se agrava o quadro fetal $^{23}$. De maneira prática podemos observar esta alteração pelo aumento da relação entre a velocidade de fluxo no ducto venoso durante a sístole ventricular e a sístole atrial (relação S/A).

Ao compararmos nossos resultados com os diponiveis na literatura podemos observar que Hecher et al., ${ }^{2,3}$ relataram associação estatisticamente significativa entre a análise de gases do feto e parâmetros de dopplervelocimetria na aorta torácica, artéria cerebral média e ducto venoso ao analisarem 23 gestações únicas, com CIUR, entre 24 e 37 semanas de gestação. Este estudo difere do nosso por utilizar a cordocentese como técnica de colheita da amostra sangüinea e por considerar como parâmetro dopplervelocimétrico para o ducto venoso o índice de pulsatilidade para veias (velocidade sistólica durante a contração atrial/média das velocidades máximas em função do tempo durante o ciclo cardíaco). 
Rizzo et $a .^{6}$ analisaram 98 fetos que apresentavam CIUR e dopplervelocimetria anormal da artéria umbilical. A colheita da amostra sangüinea também se deu por cordocentese. Após a análise dos dados por regressão logística encontraram relação estatisticamente significativa entre acidemia, hipercapnia e hipoxemia e a relação S/ A do ducto venoso. Estes mesmos autores analisaram o valor dos índices de dopplerfluxometria para ducto venoso e veia cava inferior na identificação de acidemia e hipoxemia para 209 gestações normais e 89 fetos com CIUR. Os resultados foram analisados por regressão logística e receiver operator characteristic curve (curva ROC) e observaram que para a detecção da acidemia, a veia cava inferior apresenta melhores resultados ao se considerar o índice de pré-carga (pre-load index - PLI), do que o ducto venoso (relação S/A). O PLI da veia cava inferior para detecção da acidemia apresentou sensibilidade de $73,8 \%$, e especificidade de $72,3 \%$. A relação S/A do ducto venoso para o mesmo diagnóstico apresentou sensibilidade e especificidade em torno de 72 e $60 \%$ respectivamente ${ }^{30}$.

Ao analisarmos nossos resultados podemos ver que 14 dos 48 fetos centralizados apresentaram gasometria anormal e a dopplervelocimetria do ducto venoso diagnosticou corretamente 10 destes - sensibilidade de $71 \%$. Por outro lado, 34 fetos apresentavam gasometria normal e o teste foi capaz de identificar 32 destes - especificidade de $94 \%$. Baseado nestes resultados, podemos inferir que na prematuridade extrema, antes que se considere a interrupção da gestação em face da centralização, acredita-se que a dopplervelocimetria do ducto venoso pode ajudar quando da tomada da decisão, considerando-se o risco inerente à prematuridade e ao alto custo do tratamento em unidade de terapia intensiva neonatal. Resumindo, por se tratar de teste específico, pode ser útil quando a dopplervelocimetria arterial estiver alterada.

Considerando-se o resultado da razão de verossimilhança, podemos dizer que é cerca de 12 vezes mais provável encontrar a dopplervelocimetria venosa comprometida na presença da gasometria anormal do que na ausência. A probabilidade de que a relação $\mathrm{S} / \mathrm{A}$ do ducto venoso esteja alterada na presença da gasometria anormal é de $84 \%$. A vantagem principal da razão de verossimilhança é que ela facilita ir além da simples classificação do resultado como normal ou anormal, uma vez que este será usado para a decisão de uma conduta médica.

Concordamos com Hecher et al. ${ }^{27}$ ao afirmarem que o exame dopplerfluxométrico do território venoso fetal pode ajudar na decisão do momento ideal para a interrupção da gestação em fetos centralizados. Entretanto, a idade gestacional desempenha papel importante na decisão médica, pois o risco de acidemia fetal grave precisa ser comparado ao risco da prematuridade extrema, ambos causas potenciais de mortalidade perinatal e morbidade pós-natal.

Este estudo nos permite concluir que a análise da relação $\mathrm{S} / \mathrm{A}$ do ducto venoso se mostra adequada para a predição de gasometria anormal em fetos prematuros, centralizados.

\section{ABSTRACT}

Purpose: to evaluate Doppler velocimetry of the ductus venosus as a noninvasive test of abnormal $\mathrm{pH}$ and gas analysis in preterm fetuses with "brain sparing reflex".

Methods: a cross-sectional study was performed. The studied population consisted of 48 pregnant women between the 25 th and the 33rd week of gestation, whose fetuses presented brain sparing reflex (umbilical/cerebral ratio $>1$ ). The time elapsed between Doppler velocimetry and the birth (cesarean section under peridural anesthesia) was of up to $5 \mathrm{~h}$. The following parameters were studied: $S / A$ ratio of the ductus venosus, $p H$ and base excess (BE) of fetal blood sample (collected from the umbilical vein immediately after birth). The $S / A$ ratio of the ductus venosus was considered abnormal when superior to 3.6. The fetuses were classified according to the gas analysis result. They were considered abnormal when $\mathrm{pH}$ $<7.26$ and $B E \leq 6 \mathrm{mMol} / \mathrm{L}$. Fisher's test was used for statistical analysis and considered significant when $p \leq 0.05$. Results: there was a significant correlation between umbilical blood gas analysis in preterm fetuses with brain sparing reflex and ductus venosus $S / A$ ratio $(p=0.0000082$; Fisher test). Ductus venosus Doppler velocimetry identified 10 of 14 fetuses with abnormal gas analysis. On the other hand, 32 of 34 fetuses with normal gas analysis were correctly identified. The sensitivity of the ductus venosus $S / A$ ratio for the diagnosis of abnormal blood gas analysis was 71\%, specificity 94\%, false-negative rate $8 \%$, false-positive rate $4 \%$, positive predictive value $83 \%$ and negative predictive value $89 \%$. Pretest likelihood, post-test posterior probability following a positive test result (post-test likelihood) and posttest posterior probability following a negative test result (post-test likelihood) were 31, 84 and 10\%, respectively. Conclusion: the analysis of the ductus venosus $S / A$ ratio is adequate for the diagnosis of abnormal blood gas analysis in preterm fetuses presenting brain sparing reflex.

KEYWORDS: Dopplervelocimetry. Ultrasound. Gas analysis. Fetal distress: diagnosis.

\section{Referências}

1. Cunningham FG, MacDonald PC, Gant NF, Leveno KJ, Gilstrap III LC. Preterm birth. In: Williams Obstetrics. $20^{\text {th }}$ ed. East Norwalk: Appleton \& Lange; 1997. p.797-826. 
2. Fanaroff AA, Wright LL, Stevenson DK, et al. Verylow-birth-weight outcomes of the National Institute of Child Health and Human Development Neonatal Research Network, May 1991 through December 1992. Am J Obstet Gynecol 1995; 173:1423-31.

3. Carrera JM, Pérez-Ares C, Carrera M. Bases anatómicas de la circulación maternofetal. In: Carrera J M, Montera C, Torrest M, editores. Doppler en Obstetricia. $1^{\text {a }}$ ed. Barcelona: Ed. Científicas y Tecnicas; 1992. p. 3-9.

4. Fleischer A, Schulman H, Farmakides G, et al. Uterine artery Doppler velocimetry in pregnant women with hypertension. Am J Obstet Gynecol 1986; 154:806-13.

5. Wladmiroff JW, Tonge HM, Stewart PA. Doppler ultrasound assessment of cerebral blood flow in the human fetus. Br J Obstet Gynaecol 1986; 93:471-5.

6. Rizzo G, Arduini D, Romanini C, Mancuso S. Doppler echocardiographic assessment of atrioventricular velocity waveforms in normal and small-forgestational-age fetuses. Br J Obstet Gynaecol 1988; 95:65-9.

7. Rizzo G, Arduini D. Fetal cardiac function in intrauterine growth retardation. Am J Obstet Gynecol 1991; 165:876-82.

8. Rizzo G, Arduini D, Romanini C, Mancuso S. Doppler echocardiographic assessment of time to peak velocity in the aorta and pulmonary artery of small for gestational age fetuses. Br J Obstet Gynaecol 1990; 97:603-7.

9. al-Ghazali W, Chita SK, Chapman MG, Allan LD. Evidence of redistribution of cardiac output in asymmetrical growth retardation. $\mathrm{Br} \mathrm{J}$ Obstet Gynaecol 1989; 96:697-704.

10.Hofstaetter C, Gudmundsson S, Hansmann M. Venous Doppler velocimetry in the surveillance of severely compromised fetuses. Ultrasound Obstet Gynecol 2002; 20:233-9.

11.Rizzo G, Capponi A, Arduini D, Romanini C. Ductus venosus velocity waveforms in appropriate and small for gestational age fetuses. Early Hum Dev 1994; 39:15-26.

12.Arduini D, Rizzo G, Romanini C. The development of abnormal heart rate patterns after absent enddiastolic velocity in umbilical artery: analysis of risk factors. Am J Obstet Gynecol 1993; 168:43-50.

13. Warshaw JB. Intrauterine growth retardation: adaptation or pathology? Pediatrics 1985; 76:998-9.

14.Hecher K, Snijders R, Campbell S, Nicolaides K. Fetal venous, intracardiac, and arterial blood flow measurements in intrauterine growth retardation: relationship with fetal blood gases. Am J Obstet Gynecol 1995; 173:10-5.

15.Orlandi OV, Sabrá A. O recém-nascido prematuro. In: Rezende J, editor. Obstetrícia. 9 ${ }^{a}$ ed. Rio de Janeiro: Guanabara Koogan; 2002. p.1368-77.

16.Pennati G, Bellotti M, Ferrazzi E, Rigano S, Garberi A. Hemodynamic changes across the human ductus venosus: a comparison between clinical findings and mathematical calculations. Ultrasound Obstet Gynecol 1997; 9:383-91.
17.Surerus E, Pettersen H, Penido MT, Faria M. Nos caminhos da circulação fetal: o ducto venoso. Femina 2002; 30:433-8.

18.Sá RAM, Chaves Netto H, Amim Jr J, et al. Ductus venosus velocimetry in normal pregnancy. Int $\mathrm{J}$ Gynaecol Obstet 2000; 70:28.

19.Vintzileos AM, Egan JFX, Campbell WA, et al. Asphyxia at birth as determined by cord blood $\mathrm{pH}$ measurements in preterm and term gestations: correlation with neonatal outcome. J Matern Fetal Med 1992; 1:7-13.

20.Lopes LM. Mortalidade neonatal na idade gestacional extremamente baixa (23 a 28 semanas) [tese]. Rio de Janeiro: Univ. Federal do Rio de Janeiro; 1997.

21.Baschat AA, Gembruch U, Reiss I, Gortner L, Weiner CP, Harman CR. Relationship between arterial and venous Doppler and perinatal outcome in fetal growth restriction. Ultrasound Obstet Gynecol 2000; 16:407-13.

22.Rizzo G, Capponi A, Arduini D, Romanini C. The value of fetal arterial, cardiac and venous flow in predicting $\mathrm{pH}$ and blood gases in umbilical blood at cordocentesis in growth retarded fetuses. Br J Obstet Gynaecol 1995; 102:963-9.

23.Hecher K, Campbell S. Characteristics of fetal venous blood flow under normal circumstances and during fetal disease. Ultrasound Obstet Gynecol 1996; 7:68-83.

24.Kirkpatrick SE, Pitlick PT, Naliboff J, Friedman WF. Frank-Starling relationship as an important determinant of fetal cardiac output. Am J Physiol 1976; 231:495-500.

25.Hofstaetter C, Gudmundsson S, Dubiel M, Marsál K. Ductus venosus velocimetry in high-risk pregnancies. Eur J Obstet Gynecol Reprod Biol 1996; 70:135-40.

26.Ozcan T, Sbracia M, d'Ancona RL, Copel JA, Mari G. Arterial and venous Doppler velocimetry in the severely growth-restricted fetus and associations with adverse perinatal outcome. Ultrasound Obstet Gynecol 1998; 12:39-44.

27.Hecher K, Campbell S, Snijders R, Nicolaides K. Reference ranges for fetal venous and atrioventricular blood flow parameters. Ultrasound Obstet Gynecol 1994; 4:381-90.

28. Clyman RI, Heymann MA. Fetal cardiovascular physiology. In: Creasy RK, Resnik R, editors. Maternal Fetal Medicine. $4^{\text {th }}$ ed. Philadelphia: Saunders; 1999. p.249-59.

29.Edelstone DI, Rudolph AM, Heymann MA. Effects of hypoxemia and decreasing umbilical flow liver and ductus venosus blood flows in fetal lambs. Am J Physiol 1980; 238:H656-63.

30.Rizzo G, Capponi A, Talone PE, Arduini D, Romanini C. Doppler indices from inferior vena cava and ductus venosus in predicting $\mathrm{pH}$ and oxygen tension in umbilical blood at cordocentesis in growthretarded fetuses. Ultrasound Obstet Gynecol 1996; 7:401-10.

Recebido em: 22/7/2002 Aceito com modificações em: 13/5/2003 
Laboratório: Apsen

Anúncio: Levoxin 
Laboratório: Lilly

Anúncio: Evista 